\title{
A Bayesian Approach for Parameter Estimation and Prediction Using a Computationally Intensive Model
}

\author{
Dave Higdon ${ }^{1}$, Jordan D McDonnell ${ }^{2}$, Nicolas Schunck ${ }^{2}$, Jason \\ Sarich $^{3}$, Stefan M Wild ${ }^{3}$ \\ ${ }^{1}$ Los Alamos National Laboratory, Statistical Sciences, Los Alamos, NM 87545, USA \\ 2 Physics Division, Lawrence Livermore National Laboratory, Livermore, CA 94551, \\ USA \\ ${ }^{3}$ Mathematics and Computer Science Division, Argonne National Laboratory, \\ Argonne, IL 60439, USA \\ E-mail: dhigdon@lanl.gov
}

\begin{abstract}
Bayesian methods have been successful in quantifying uncertainty in physics-based problems in parameter estimation and prediction. In these cases, physical measurements $y$ are modeled as the best fit of a physics-based model $\eta(\theta)$, where $\theta$ denotes the uncertain, best input setting. Hence the statistical model is of the form

$$
y=\eta(\theta)+\epsilon,
$$

where $\epsilon$ accounts for measurement, and possibly other, error sources. When nonlinearity is present in $\eta(\cdot)$, the resulting posterior distribution for the unknown parameters in the Bayesian formulation is typically complex and nonstandard, requiring computationally demanding computational approaches such as Markov chain Monte Carlo (MCMC) to produce multivariate draws from the posterior. Although generally applicable, MCMC requires thousands (or even millions) of evaluations of the physics model $\eta(\cdot)$. This requirement is problematic if the model takes hours or days to evaluate. To overcome this computational bottleneck, we present an approach adapted from Bayesian model calibration. This approach combines output from an ensemble of computational model runs with physical measurements, within a statistical formulation, to carry out inference. A key component of this approach is a statistical response surface, or emulator, estimated from the ensemble of model runs. We demonstrate this approach with a case study in estimating parameters for a density functional theory model, using experimental mass/binding energy measurements from a collection of atomic nuclei. We also demonstrate how this approach produces uncertainties in predictions for recent mass measurements obtained at Argonne National Laboratory.
\end{abstract}

PACS numbers: 21.10.-k, 21.30.Fe, 21.60.Jz, 21.65.Mn

Submitted to: J. Phys. G: Nucl. Phys. 


\section{Introduction}

Bayesian calibration of computer models [1, 2, 3] combines output from an ensemble of computational model runs with physical measurements, within a statistical formulation, in order to carry out statistical and scientific inferences. These inferences include quantifying the uncertainty in model parameters as well as in model-based predictions. Unlike more standard Bayesian inverse methodologies [4, 5], model calibration approaches must accommodate the high cost of evaluating the computational model; in many cases, only a limited number of model evaluations can be made. Also, because the model can never exactly match the physical measurements, even at the best possible parameter input settings, the statistical formulation must account for this discrepancy.

Since the computational model cannot be quickly evaluated at any input setting when needed, an ensemble of model runs is carried out prior to the statistical analysis, producing the raw material from which to build a response surface, mapping the model parameter inputs to the model outputs of interest. A Gaussian process (GP) model is most commonly used to emulate the model response as a function of the inputs $[6,7]$. Not only does the GP model typically produce an accurate emulator [8], it can also be embedded within a statistical formulation, allowing parameter estimation (calibration) and model-based prediction.

In this paper, we apply the Bayesian model calibration approach to the nuclear density functional theory (DFT) model described by Schunck et al. [9] in this issue, as well as in other references $[10,11]$. To streamline the presentation, we focus on nuclear masses for a number of spherical and deformed nuclei as the quantities of interest for both the model and the experimental measurements. Our goal is to estimate parameter uncertainties from this data and to produce predictions, with uncertainty, for a recent set of mass measurements carried out at the CARIBU facility at Argonne National Laboratory (ANL) [12].

In the following sections, we briefly review the experimental measurements and DFT model before describing the statistical formulation in greater detail. We apply the Bayesian model calibration approach to this data, producing updated uncertainties for the model parameters and prediction uncertainties for the new ANL mass measurements, and we compare these predictions with the experimental measurements. We end with a discussion of this approach, pointing out features of this analysis and describing its strengths and weakness.

\subsection{Bayesian Formulations}

Before presenting the Bayesian formulation used for this case study, we give a brief overview of Bayesian approaches for parameter estimation, focusing on a simple, 1-d example shown in Figure 1. Physical data $y$ is combined with a scientifically motivated model $\eta(\cdot)$. The model requires an input parameter $\theta$ to make a prediction $\hat{y}=\eta(\theta)$. The goal is to use the data $y$ to constrain uncertainty regarding $\theta$. Uncertainty in $\theta$ then induces uncertainty in a new prediction $\eta(\theta)$. 


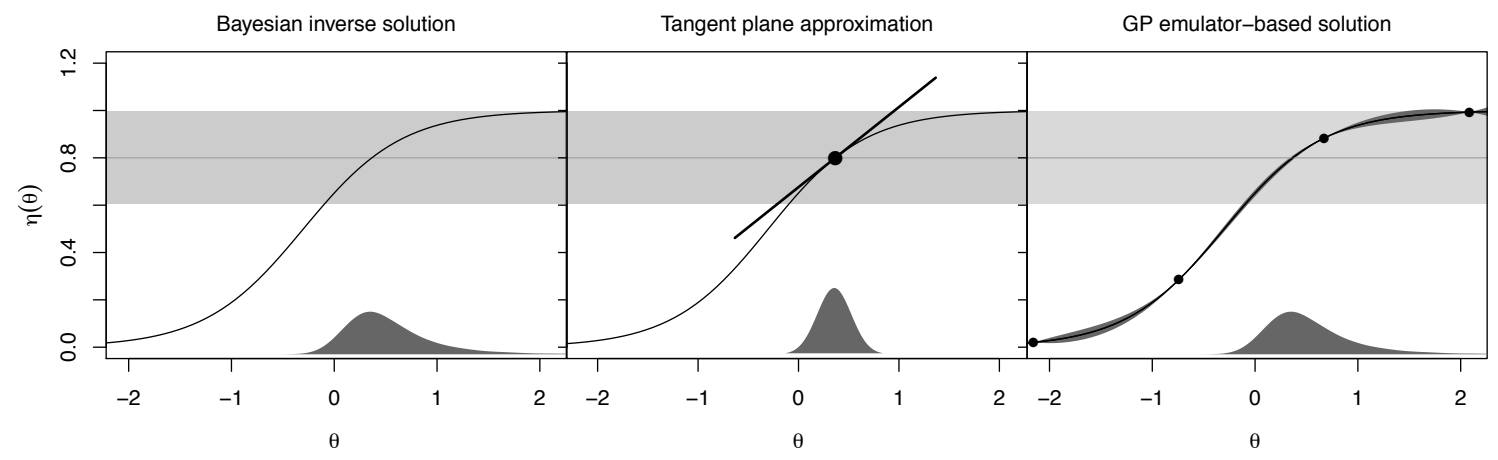

Figure 1. Possible Bayesian approximations to a 1-d estimation problem. The horizontal line represents the observation of $y=.8$, whose error has a standard deviation of $\sigma=.1$; the $\pm 2 \sigma$ region is represented by the shaded region about the line. The sigmoid line shows the model $\eta(\theta)$, and the shaded density on the $x$-axis shows the posterior distribution for $\theta$. Left: the exact posterior density for $\theta$ is estimated via MCMC; middle: a linear tangent approximation to the model $\eta(\cdot)$ is used to induce a normal approximation to the posterior density for $\theta$. Right: a GP is used to estimate $\eta(\cdot)$ using 4 model runs (black dots), producing a more accurate approximation to the posterior density for $\theta$.

The Bayesian paradigm requires specification of the likelihood $L(y \mid \theta)$, and the prior for the unknown parameter $\pi(\theta)$. The inference is based on the posterior distribution, whose (unnormalized) density is just the product of the likelihood and the prior

$$
\pi(\theta \mid y) \propto L(y \mid \theta) \times \pi(\theta)
$$

In the simple example of Figure 1, the physical observation $y$ is modeled as

$$
y=\eta(\theta)+\epsilon, \text { where } \epsilon \sim N\left(0,0.1^{2}\right)
$$

where $\sim$ means "is distributed as." Thus $L(y \mid \theta) \propto \exp \left\{-\frac{1}{2} \cdot 0.1^{-2}(y-\eta(\theta))^{2}\right\}$. For the prior we take $\theta \sim N(0,1)$ so that $\pi(\theta) \propto \exp \left\{-\frac{1}{2} \theta^{2}\right\}$. Thus the resulting posterior density for $\theta$ is given by

$$
\pi(\theta \mid y) \propto \exp \left\{-\frac{1}{2}\left[0.1^{-2}(y-\eta(\theta))^{2}+\theta^{2}\right]\right\} .
$$

Bayesian inference requires understanding this resulting posterior distribution and using it to make predictions for new observations $y$. While the 1-d example shown here is not too daunting, typical examples have a much higher-dimensional parameter space, including statistical nuisance parameters such as variances. In such cases, Markov chain Monte Carlo (MCMC) [13] can be used to generate a realization from an ergodic Markov chain, producing a (dependent) sequence of samples $\left\{\theta^{(1)}, \ldots, \theta^{(T)}\right\}$ from the posterior distribution $\pi(\theta \mid y)$. This Markov chain is most commonly produced using some form of rejection step, where the transition from $\theta^{(k)}$ to $\theta^{(k+1)}$ is taken by proposing a new value $\theta^{*}$, and setting $\theta^{(k+1)}$ to either $\theta^{*}$ or $\theta^{(k)}$ with a probability that depends on the ratio $\pi\left(\theta^{*} \mid y\right) / \pi\left(\theta^{(k)} \mid y\right)$. Hence, producing a useful sample from $\pi(\theta \mid y)$ with MCMC requires 
many thousands to millions of evaluations of $\eta(\theta)$. In our case study, $\eta(\theta)$ represents a computationally demanding DFT model, making direct use of MCMC infeasible. A common alternative is to replace the computational model $\eta(\theta)$ by a linear tangent model approximation. While easy to work with, this linear assumption often results in overly narrow estimates of the parameter uncertainty (see the middle frame of Figure 1). In this paper we develop a Gaussian process (GP)-based approximation for $\eta(\cdot)$, allowing posterior sampling with MCMC, without additional evaluations of $\eta(\theta)$.

\subsection{DFT Model and Experimental Measurements}

The DFT model considered in this case study is based on the UNEDF1 parametrization of the Skyrme functional [11]. It requires a $p=12$-dimensional parameter vector $t=\left(t_{1}, \ldots, t_{p}\right)$ to produce fitted masses for any given nucleus. The parameters and their prior ranges are given in Table 1 . For any input setting $t$ and any specified nucleus $(N, Z)$, the DFT code produces a fitted mass. Note that we use $t$ to denote a generic setting of the inputs, reserving $\theta$ to denote the unknown best input setting, which is to be estimated from the experimental mass measurements.

Table 1. DFT model parameters for the UNEDF1 parametrization and their prior ranges

\begin{tabular}{cccc}
\hline \hline Parameter & Label & $C_{\text {lower }}$ & $C_{\text {upper }}$ \\
\hline$\rho_{c}$ & $\theta_{1}$ & 0.155 & 0.165 \\
$E^{N M} / A$ & $\theta_{2}$ & -16.0 & -15.5 \\
$K^{N M}$ & $\theta_{3}$ & 200 & 240 \\
$a_{\mathrm{sym}}^{N M}$ & $\theta_{4}$ & 27.0 & 31.0 \\
$L_{\mathrm{sym}}^{N M}$ & $\theta_{5}$ & 15.0 & 65.0 \\
$1 / M_{s}^{*}$ & $\theta_{6}$ & 0.75 & 1.25 \\
$C_{0}^{\rho \Delta \rho}$ & $\theta_{7}$ & -60 & -30 \\
$C_{1}^{\rho \Delta \rho}$ & $\theta_{8}$ & -240 & -50 \\
$V_{0}^{n}$ & $\theta_{9}$ & -220 & -150 \\
$V_{0}^{p}$ & $\theta_{10}$ & -230 & -180 \\
$C_{0}^{\rho \nabla J}$ & $\theta_{11}$ & -90 & -60 \\
$C_{1}^{\rho \nabla J}$ & $\theta_{12}$ & -90 & 20 \\
\hline \hline
\end{tabular}

The example illustrated in Figure 2 considers the masses of the 28 spherical nuclei and 47 deformed nuclei included in the original UNEDF1 parametrization, supplemented by the masses of 17 neutron-rich nuclei recently measured at the CARIBU facility at Argonne National Laboratory [12]. For a given input setting $t$, the DFT code is run to compute the masses for all $n=n_{1}+n_{2}+n_{3}=28+47+17=92$ nuclei, producing an $n$-vector of outputs $\eta(t)$. For the Bayesian analysis, we generate an initial set of $m=183$ DFT model runs for each of these $n$ nuclei. Note that the original design was for 200 parameter settings, but 17 of these runs were discarded because of convergence 
issues. The output range of the resulting $n \times m=16,836$ DFT runs are shown in Figure 2 for each of the 92 nuclei. The input settings used in this analysis are shown in Figure 3 .

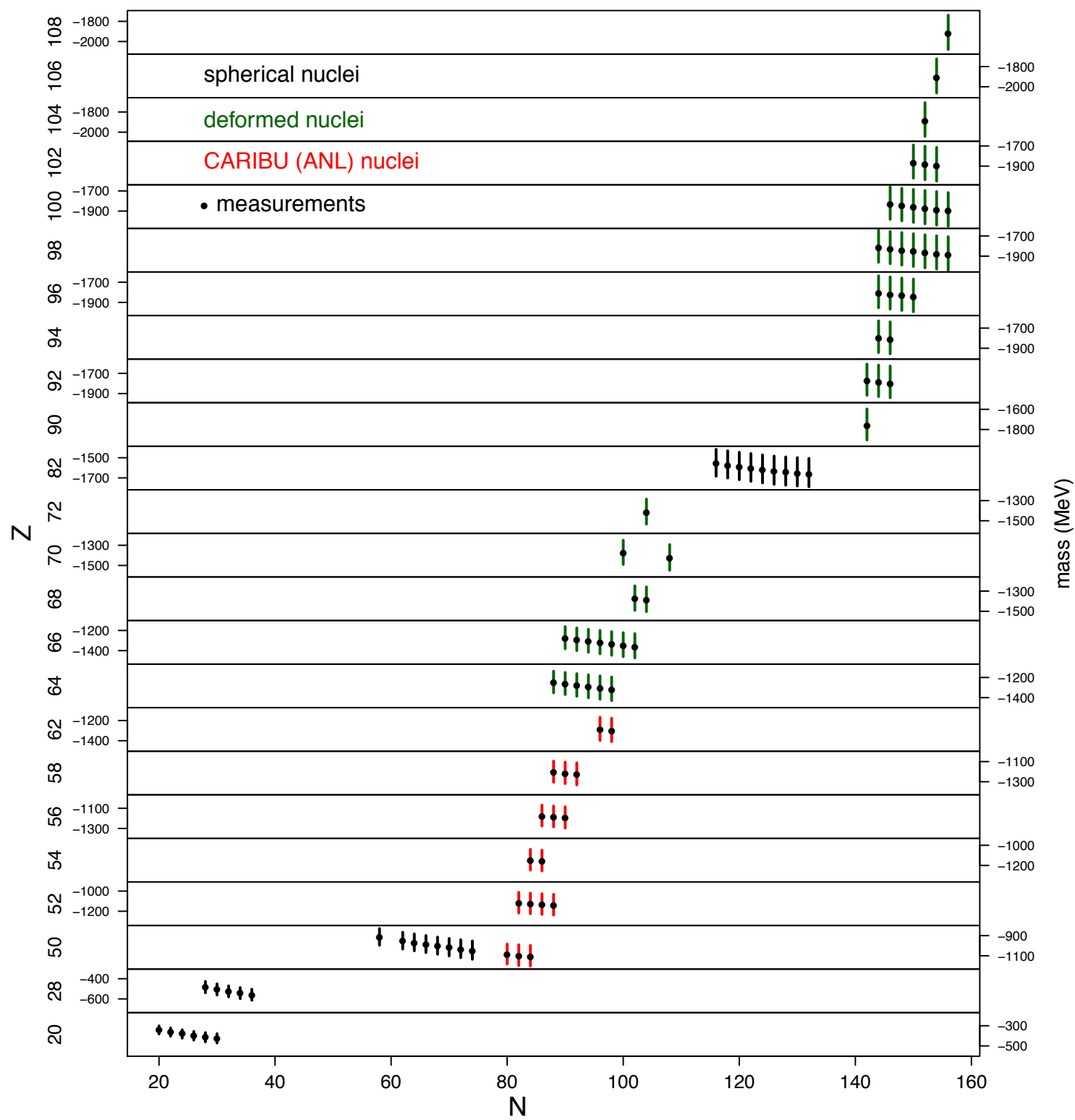

Figure 2. Range of DFT computed masses (vertical lines), along with the experimental measurements (dots) for the 28 spherical (black) and 47 deformed (green) nuclei of the original UNEDF1 parametrization, as well as the 17 newly measured neutron-rich nuclei (red). The ranges of DFT computed masses are derived from the ensemble of 183 parameter settings shown in Figure 3.

In addition to the model runs, each nucleus has an experimentally determined mass denoted by the black dot in Figure 2. As a demonstration of this Bayesian model calibration methodology, we use the data from the 28 spherical and 47 deformed nuclei of UNEDF1 to constrain model parameter uncertainties and to estimate model error. 
With these results, we compare the resulting predictions, and their uncertainties, with the actual measurements of the 17 neutron-rich nuclei obtained at ANL [12].

\section{Bayesian Formulation}

The full Bayesian model formulation combines experimental measurements and an ensemble of DFT model runs, all within an encompassing statistical model. Hence we describe the main components of this model in the next three subsections. The first component, described in Section 2.1, is the GP model used to probabilistically describe the DFT output given parameter settings $t$. The second component, described in Section 2.3, is a full Bayesian model calibration formulation for a single output type (spherical masses), using the simulation output, along with the experimental measurements to reduce parameter uncertainties. The third component, described in Section 2.4, combines separate formulations into a single Bayesian model, so that information from multiple data types can be used to estimate parameter uncertainties both for the DFT model and for statistical parameters that control the GP covariance and the error variance.

Once the various parameter uncertainties are estimated within this overarching statistical model, predictions and predictive distributions for outputs of interest can be determined. We will compare the predictive distribution with each of the experimental measurements used for estimation (i.e., the masses for the spherical and deformed nuclei). We will also compare predictive distributions for the new ANL mass measurements for neutron-rich nuclei. Since the ANL mass measurements were not used to estimate any of the model parameters, we can assess the quality of the predictions produced by this statistical formulation.

\subsection{Emulation of DFT Model Output}

The DFT model requires 5-10 minutes to compute the mass for a given nucleus. Given that the statistical analysis presented here involves 75 nuclei, requiring 75 DFT solves for every evaluation of $\eta(\theta)$, direct use of MCMC is not practical. In this case study, therefore, we treat $\eta(\cdot)$ as an unknown function, to be estimated from an initial set of $m=183$ DFT solves for each nucleus. In this section we describe a Bayesian approach for estimating $\eta(\cdot)$ from the $m$ training runs with a Gaussian process (GP) specification. A Bayesian approach is taken that can be integrated into the full model formulation.

The use of a GP model to emulate a computational model $\eta(\cdot)$ at new parameter inputs dates back $25+$ years $[6,14]$. The approach has proven effective in various applications where the model output changes smoothly as a function of the inputs $\theta$ $[15,16,17]$.

The Ensemble of DFT Runs. We start with simulator runs at $m$ different input settings

$$
\eta\left(t_{j}^{*}\right), j=1, \ldots, m .
$$


We use a space-filling Latin hypercube [18] sample for this initial design of input settings $\left(t_{1}^{*}, \ldots, t_{m}^{*}\right)$. We use $\boldsymbol{t}^{*}$ to denote the $m \times p$ matrix describing this design, or ensemble of input settings. Two-dimensional projections of this parameter design are shown in Figure 3. How best to construct a design for an emulator is still a research topic in the statistical literature; a starting point to this literature can be found in Santer et al.'s textbook [19] and the references therein.

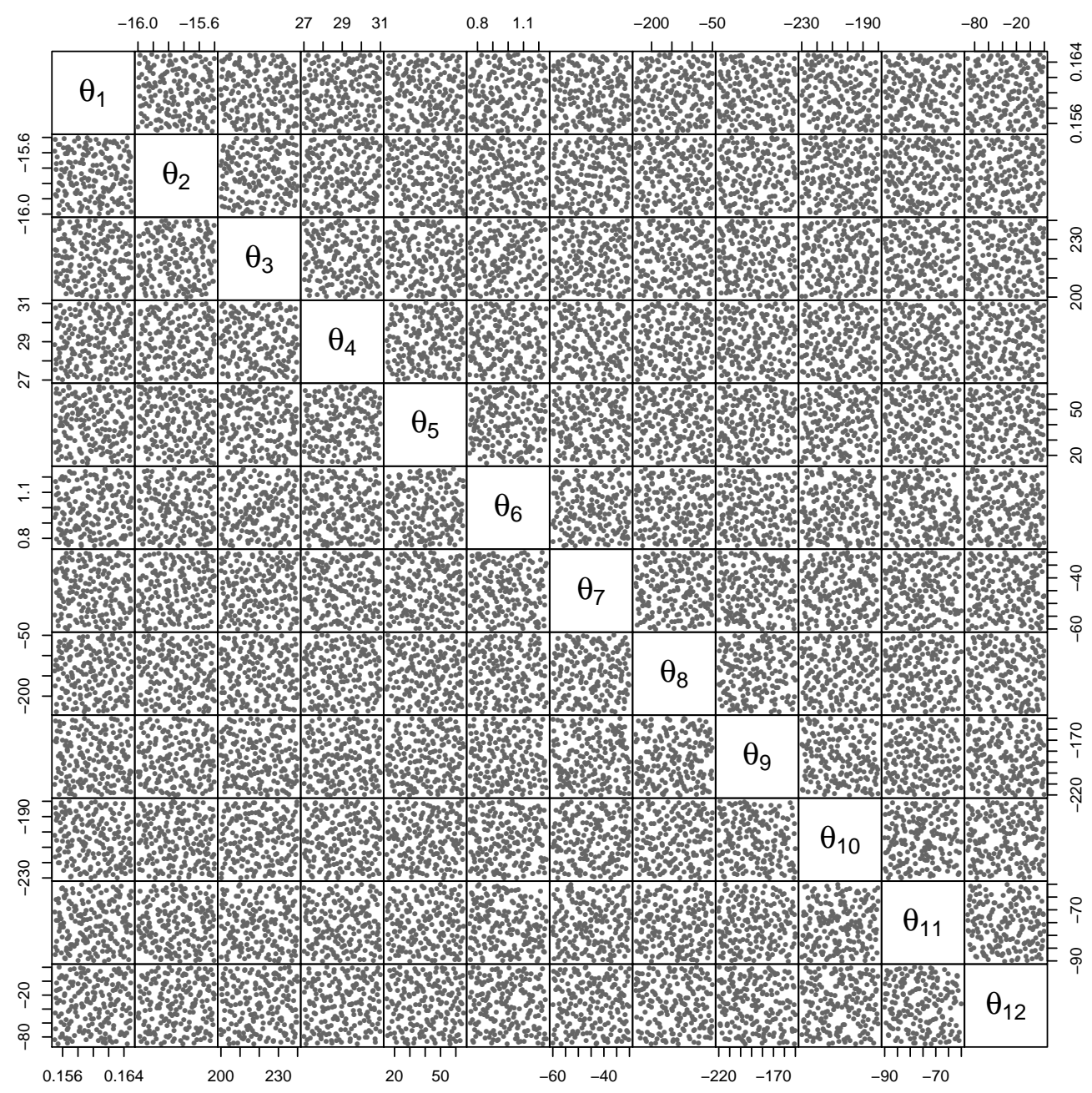

Figure 3. Space-filling Latin hypercube sample (LHS) used to specify the initial set of DFT runs with which to construct the GP emulator. The 2-d projections of this design are shown for each pair of parameters.

\subsection{Basis Representation of the DFT Model Output}

We focus here on a single output type: the $n=28$ masses for the spherical nuclei. There are a number of approaches available for extending the univariate GP model to handle many outputs at once $[20,21]$; we use the basis approach of [22] which performs well in this application. 
To simplify the model specification, we standardize the $p=12$-dimensional parameter space to $C=[0,1]^{p}$ using the ranges given in Table 1 . For a given input $t$ in the standardized input space $[0,1]^{p}$, the DFT model produces an $n$-vector $\eta(t)$, giving the mass for each of the $n$ nuclei. The emulator models the DFT output using a $q$-dimensional basis representation:

$$
\eta(t)=\sum_{i=1}^{q} \phi_{i} w_{i}(t)+e, t \in[0,1]^{p},
$$

where $\left\{\phi_{1}, \ldots, \phi_{q}\right\}$ are orthogonal, $n$-dimensional basis vectors, the $w_{i}(t)$ are weights whose value depends on $t$, and $e$ is an $n$-dimensional error term, accounting for the residual in the basis representation, as well as numerical noise - associated with finiteprecision (e.g., roundoff error) and finite-process (e.g., residual tolerances, adaptive discretizations) computations - from the DFT code. This formulation builds an emulator that maps $[0,1]^{p}$ to $R^{n}$ by building $q$ independent, univariate models for each $w_{i}(t)$. Separate Gaussian processes models $[6,22]$ are used to model each of the weight functions; this is described in the following two subsections.

For each of the $m=183$ settings $\left(t_{1}^{*}, \ldots, t_{m}^{*}\right)$ of parameter design, an $n$-dimensional vector of masses is produced, giving $\eta_{1}, \ldots, \eta_{m}$. These output vectors are represented by principal components [23] or, equivalently, by empirical orthogonal functions (EOFs) [24]. Following standard practice, the output vectors are centered by subtracting the mean $\left(\frac{1}{m} \sum_{j=1}^{m} \eta_{j}\right)$ from each output vector. Alternative standardizations may be preferred, depending on the application. This same standardization is also applied to the experimental data.

We obtain the $n \times m$ matrix $\Xi$ by column-binding the (standardized) output vectors from the simulations. Applying a singular value decomposition (SVD) to the simulation output matrix $\Xi$ gives

$$
\Xi=\left[\eta_{1} ; \cdots ; \eta_{m}\right]=U D V^{\prime},
$$

where $U$ is a $n \times m$ orthogonal matrix, $D$ is a diagonal $m \times m$ matrix holding the singular values, and $V$ is a $m \times m$ orthonormal matrix. To construct a $q$-dimensional representation of the simulation output, we define the EOF basis matrix $\Phi_{\eta}$ to be the first $q$ columns of $[U D \sqrt{m}]$. We take $q=9$; this is sufficient to explain over $99.9 \%$ of the variation in the simulation ensemble. For illustration, the first three basis functions $\phi_{1}, \ldots, \phi_{3}$ are shown in Figure 4 . Note that the $\phi_{i}$ are $n=28$-dimensional vectors, with one element for each spherical nucleus.

2.2.1. Specifying the Gaussian Process Model Emulator. Each of the basis weights in Equation $(1), w_{i}(t), i=1, \ldots, q$, is a function mapping the $p$-dimensional input $t$ to a scalar. These functions are modeled (a priori) as independent, mean zero GPs,

$$
w_{i}(\cdot) \sim \operatorname{GP}(\mu(\cdot), C(\cdot, \cdot)) \text { with } \mu(t)=0, C\left(t, t^{\prime}\right)=\lambda_{w i}^{-1} R\left(t, t^{\prime} ; \rho_{i}\right),
$$

where $\lambda_{w i}$ is the marginal precision (precision $=1$ /variance) of the process and $R\left(t, t^{\prime} ; \rho_{i}\right)$ is a correlation function, whose entries depend on the pair of input settings $t$ and $t^{\prime}$, as 


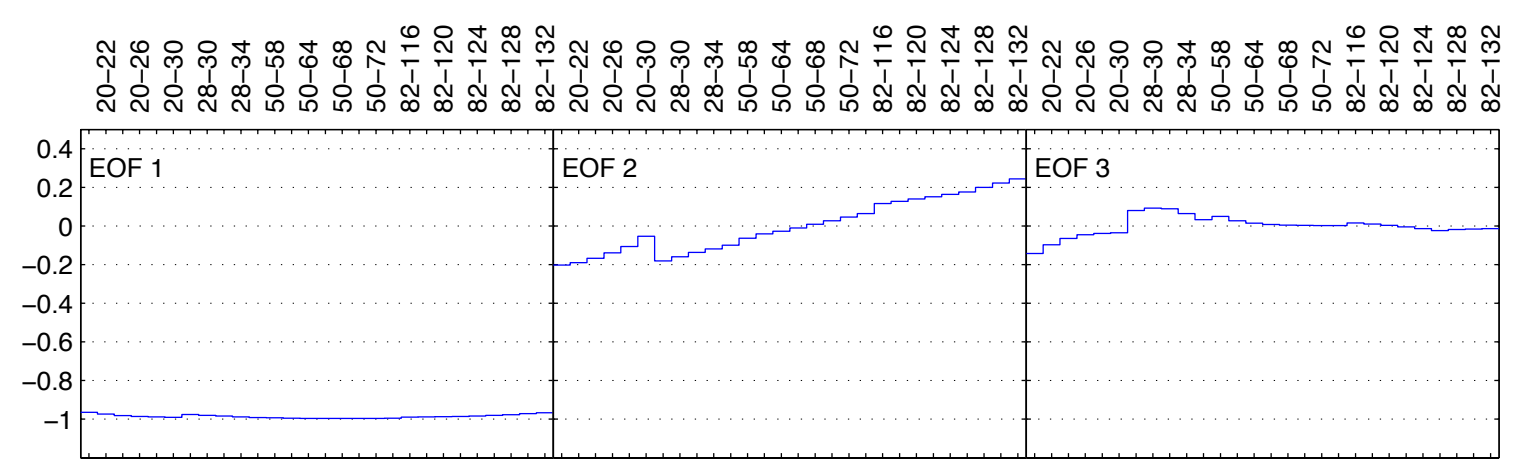

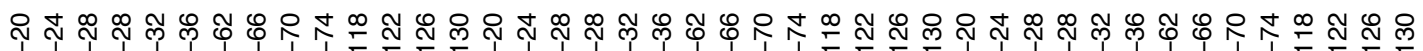

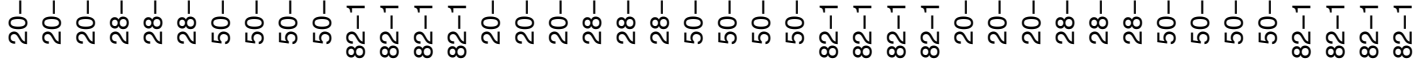

Figure 4. The first three basis functions $\phi_{i}, i=1, \ldots, 3$, over each of the 28 spherical nuclei. Here each of the basis functions is derived by using empirical orthogonal functions from the 183 simulations. Each nucleus is labeled $Z-N$.

well as the vector $\rho_{i}=\left(\rho_{i 1}, \ldots, \rho_{i p}\right)^{\prime}$ :

$$
R\left(t, t^{\prime} ; \rho_{i}\right)=\prod_{k=1}^{p} \rho_{i k}^{4\left(t_{k}-t_{k}^{\prime}\right)^{2}} .
$$

This is the Gaussian covariance function, giving smooth realizations, and commonly used to model computer simulation output $[1,6]$. This fits well with our expectation that the DFT masses change smoothly as the input values are changed. An advantage of the product form is that only a single additional parameter is required per additional input dimension, while the fitted GP response still allows for general interactions between inputs. The parameter $\rho_{i k}$ controls the spatial range for the $k$ th input dimension of the process $w_{i}(\cdot)$. Under this parameterization, $\rho_{i k}$ gives the correlation between $w_{i}(t)$ and $w_{i}\left(t^{\prime}\right)$ when the input conditions $t$ and $t^{\prime}$ are identical, except for a difference of 0.5 in the $k$ th component. Note that this interpretation uses the standardization of the input space to $[0,1]^{p}$.

Restricting our attention to the $m$ input design settings, we define the $m$-vector $w_{i}$ to be $w_{i}=\left(w_{i}\left(t_{1}^{*}\right), \ldots, w_{i}\left(t_{m}^{*}\right)\right)^{\prime}$ for $i=1, \ldots, q$. In addition we define $R\left(\boldsymbol{t}^{*} ; \rho_{i}\right)$ to be the $m \times m$ correlation matrix resulting from applying Equation (4) to each pair of input settings in the design $\boldsymbol{t}^{*}$. The $p$-vector $\rho_{i}$ gives the correlation distances for each of the input dimensions. At the $m$ simulation input settings, the $m q$-vector $w=\left(w_{1}^{\prime}, \ldots, w_{q}^{\prime}\right)^{\prime}$ then has prior distribution

$$
\left(\begin{array}{c}
w_{1} \\
\vdots \\
w_{q}
\end{array}\right) \sim N\left(\left(\begin{array}{c}
0 \\
\vdots \\
0
\end{array}\right),\left(\begin{array}{ccc}
\lambda_{w 1}^{-1} R\left(\boldsymbol{t}^{*} ; \rho_{1}\right) & 0 & 0 \\
0 & \ddots & 0 \\
0 & 0 & \lambda_{w q}^{-1} R\left(\boldsymbol{t}^{*} ; \rho_{q}\right)
\end{array}\right)\right),
$$

which is controlled by $q$ precision parameters held in $\lambda_{w}$ and $q \cdot p$ spatial correlation parameters held in $\rho$. The prior above can be written more compactly as $w \sim N\left(0, \Sigma_{w}\right)$, where $\Sigma_{w}$, controlled by parameter vectors $\lambda_{w}$ and $\rho$, is given by the block diagonal covariance matrix in Equation (5). 
2.2.2. Bayesian Representation of the GP Emulator. We specify independent gamma priors $G a\left(a_{w}, b_{w}\right)$ for each $\lambda_{w i}$ and independent beta priors for the $\rho_{i k}$, giving the prior densities

$$
\begin{aligned}
& \pi\left(\lambda_{w i}\right) \propto \lambda_{w i}^{a_{w}-1} e^{-b_{w} \lambda_{w i}}, \quad i=1, \ldots, q \\
& \pi\left(\rho_{i k}\right) \propto \rho_{i k}^{a_{\rho}-1}\left(1-\rho_{i k}\right)^{b_{\rho}-1}, \quad i=1, \ldots, q, k=1, \ldots, p .
\end{aligned}
$$

We expect the marginal variance for each $w_{i}(\cdot)$ process to be close to one, because of the scaling of the basis functions. For this reason we specify that $a_{w}=b_{w}=5$, encouraging each $\lambda_{w i}$ to be close to 1 . In addition, this informative prior helps stabilize the resulting posterior distribution for the correlation parameters that can trade off with the marginal precision parameter. Because we expect only a subset of the inputs to influence the simulator response, our prior for the correlation parameters reflects this expectation of effect sparsity for each $w_{i}(\cdot)$. Under the parameterization in Equation (4), input $k$ is inactive for PC $i$ if $\rho_{i k}=1$. Choosing $a_{\rho}=1$ and $0<b_{\rho}<1$ yields a density with substantial prior mass near 1 . We take $b_{\rho}=0.1$, which makes $\operatorname{Pr}\left(\rho_{i k}<0.98\right) \approx \frac{1}{3}$ a priori. In general, the selection of these hyperparameters should depend on how many of the $p$ inputs are expected to be active.

We can now define the likelihood, or sampling model, for the simulation output $\eta$. Here $\eta=\operatorname{vec}(\Xi)$, where $\operatorname{vec}(\Xi)$ produces a vector by stacking the columns of matrix $\Xi$. Taking the error vector in Equation (1) to be independent Gaussian with common precision $\lambda_{\eta}$, we get the sampling model, or likelihood, for $\eta$ :

$$
\eta \mid w, \lambda_{\eta} \sim N\left(\Phi w, \lambda_{\eta}^{-1} I\right)
$$

where $\Phi=\left[I_{m} \otimes \phi_{1} ; \cdots ; I_{m} \otimes \phi_{q}\right]$ and the $\phi_{i}$ are the $q$ basis vectors previously computed by SVD. A $G a\left(a_{\eta}, b_{\eta}\right)$ is specified for the error precision $\lambda_{\eta}$.

Multiplying the probability density functions implied by Equations (5), (6), and (7) and the gamma prior for $\lambda_{\eta}$ yields the (unnormalized) posterior density. After integrating out $w$, the posterior distribution for the unknown parameters becomes

$$
\begin{aligned}
& \pi\left(\lambda_{\eta}, \lambda_{w}, \rho \mid \eta\right) \propto \\
& \left|\left(\lambda_{\eta} \Phi^{\prime} \Phi\right)^{-1}+\Sigma_{w}\right|^{-\frac{1}{2}} \exp \left\{-\frac{1}{2} \hat{w}^{\prime}\left(\left[\lambda_{\eta} \Phi^{\prime} \Phi\right]^{-1}+\Sigma_{w}\right)^{-1} \hat{w}\right\} \times \\
& \lambda_{\eta}^{a_{\eta}^{*}-1} e^{-b_{\eta}^{*} \lambda_{\eta}} \times \prod_{i=1}^{q} \lambda_{w i}^{a_{w}-1} e^{-b_{w} \lambda_{w i}} \times \prod_{i=1}^{q} \prod_{j=1}^{p}\left(1-\rho_{i j}\right)^{b_{\rho}-1}
\end{aligned}
$$

where

$$
\begin{aligned}
& a_{\eta}^{*}=a_{\eta}+\frac{m(n-q)}{2}, \\
& b_{\eta}^{*}=b_{\eta}+\frac{1}{2} \eta^{\prime}\left(I-\Phi\left(\Phi^{\prime} \Phi\right)^{-1} \Phi^{\prime}\right) \eta, \text { and } \\
& \hat{w}=\left(\Phi^{\prime} \Phi\right)^{-1} \Phi^{\prime} \eta .
\end{aligned}
$$

In some applications $\hat{w}$ - the simulation output dotted with the basis vectors - may not exactly conform to a smooth response over the input space. This situation is often due to numerical jitter in the computational model. In such cases, an additional error 
may be required. We typically add the $m q \times m q$ covariance matrix $\operatorname{diag}\left(\lambda_{o 1} I_{m}, \ldots, \lambda_{o q} I_{m}\right.$ to the covariance term in (8) to allow for some mismatch between $\hat{w}_{i}$ and $w\left(t_{i}\right)$. In this case, independent $G a(1, .0001)$ priors are used for the $\lambda_{o i}$ 's.

\subsubsection{Exploring the Posterior Distribution for the Emulator. The posterior} distribution is a necessary ingredient for the complete formulation that incorporates the experimental data. However, it is often also worth exploring this intermediate posterior distribution for the DFT model response. For this purpose we use MCMC and standard Metropolis updates [25, 13] and we view a number of posterior quantities to illuminate features of the DFT model. The posterior of the emulator response can be used to investigate sensitivity measures of computational model [7] or to estimate a Sobol decomposition of the model response [6]. Figure 5 shows boxplots of the posterior distributions for the components of $\rho$. From this figure it is apparent that the PC's are influenced by a number of the components in $t$. Figure 6 shows the resulting posterior mean surfaces for $w_{1}(\cdot), w_{2}(\cdot)$, and $w_{3}(\cdot)$ as a function of $t_{6}$ and $t_{7}$.

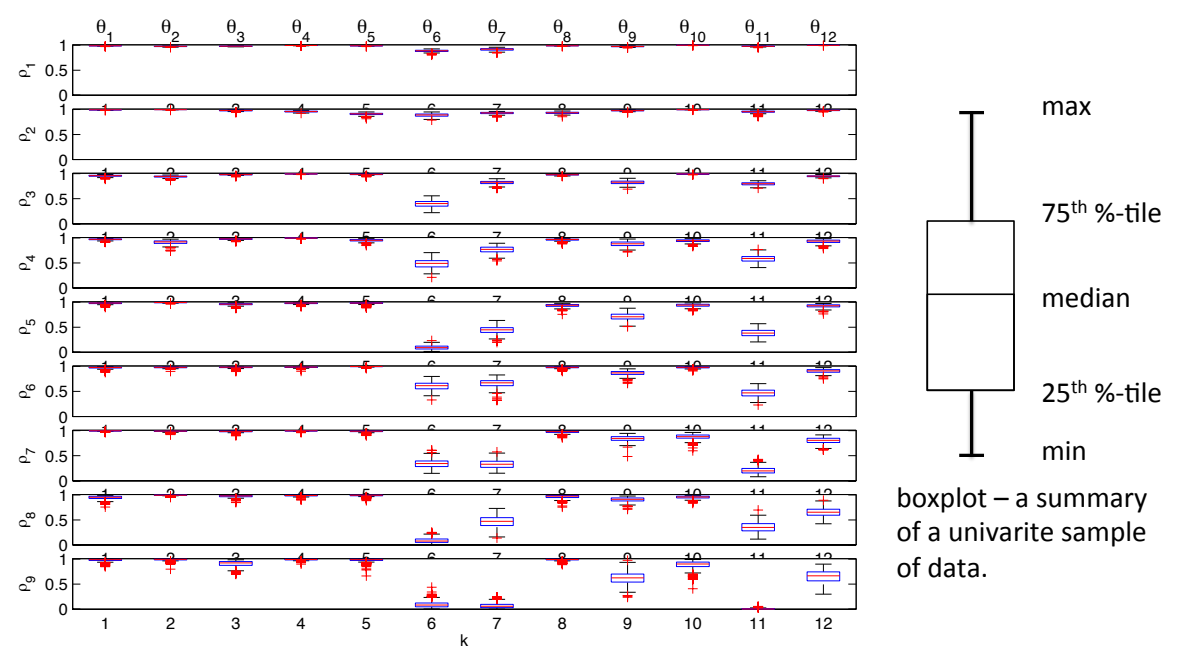

Figure 5. Boxplots of posterior samples for each $\rho_{i k}$, which control the GP response surface that predicts mass for the spherical nuclei as a function of the 12 DFT parameters.

2.2.4. Generating Emulator-Based Predictions. Given the posterior realizations from Equation (8), one can generate realizations from the process $\eta(\cdot)$ at any input setting $t^{\star}$. Since

$$
\eta\left(t^{\star}\right)=\sum_{i=1}^{q} \phi_{i} w_{i}\left(t^{\star}\right),
$$

realizations from the $w_{i}\left(t^{\star}\right)$ processes need to be drawn given the MCMC output. For a given draw $\left(\lambda_{\eta}, \lambda_{w}, \rho\right)$ a draw of $w^{\star}=\left(w_{1}\left(t^{\star}\right), \ldots, w_{q}\left(t^{\star}\right)\right)^{\prime}$ can be produced by using 


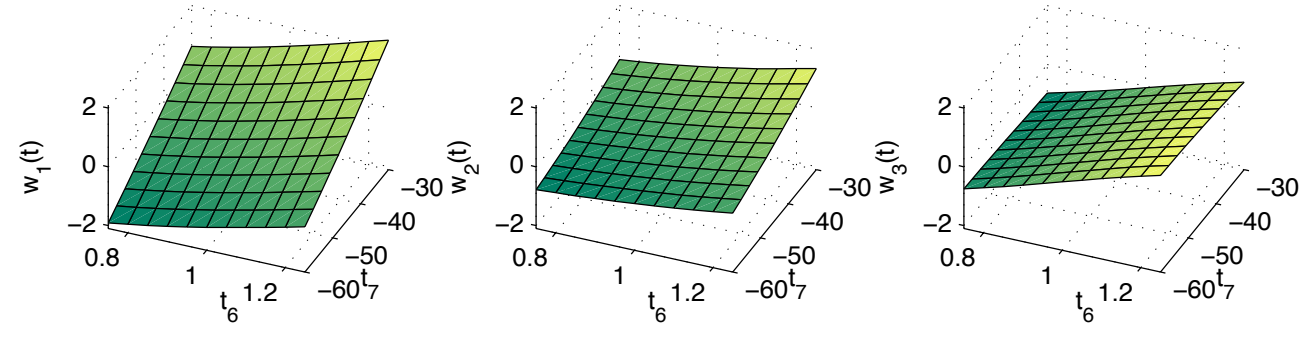

Figure 6. Posterior mean surfaces for $w_{i}(\cdot), i=1,2,3$, the weights corresponding to the first three EOF's. Here the remaining 10 parameters were held at their midpoints as $t_{6}$ and $t_{7}$ vary over their design range.

the fact

$$
\left(\begin{array}{c}
\hat{w} \\
w^{\star}
\end{array}\right) \sim N\left(\left(\begin{array}{l}
0 \\
0
\end{array}\right),\left[\left(\begin{array}{cc}
\left(\lambda_{\eta} \Phi^{\prime} \Phi\right)^{-1} & 0 \\
0 & 0
\end{array}\right)+\Sigma_{w, w^{\star}}\left(\lambda_{w}, \rho\right)\right]\right),
$$

where $\Sigma_{w, w^{\star}}$ is obtained by applying the covariance rule from Equation (4) to the augmented input settings that include the original design $\boldsymbol{t}$ and the new input setting $t^{\star}$. Recall that $\hat{w}=\left(\Phi^{\prime} \Phi\right)^{-1} \Phi^{\prime} \eta$. Application of the conditional normal rules then gives

$$
w^{\star} \mid \hat{w} \sim N\left(V_{21} V_{11}^{-1} \hat{w}, V_{22}-V_{21} V_{11}^{-1} V_{12}\right),
$$

where

$$
V=\left(\begin{array}{ll}
V_{11} & V_{12} \\
V_{21} & V_{22}
\end{array}\right)=\left[\left(\begin{array}{cc}
\left(\lambda_{\eta} \Phi^{\prime} \Phi\right)^{-1} & 0 \\
0 & 0
\end{array}\right)+\Sigma_{w, w^{\star}}\left(\lambda_{w}, \rho\right)\right]
$$

is a function of the parameters produced by the MCMC output. Hence, for each posterior realization of $\left(\lambda_{\eta}, \lambda_{w}, \rho\right)$, a realization of $w^{\star}$ can be produced. This approach easily generalizes to give predictions over many input settings at once.

Figure 7 shows posterior means for the simulator response $\eta(\cdot)$ where each of the inputs is varied over its prior (standardized) range of $[0,1]$ while the other 11 inputs are held at their midpoints.

The posterior mean response conveys an idea of how the different parameters affect the computed masses for these three nuclei. The sensitivities for other spherical, deformed, and neutron-rich ANL masses are similar. Other marginal functionals of the simulation response can also be calculated, such as sensitivity indices or estimates of the Sobol decomposition [6, 7].

Note that a simpler emulator could have been constructed by estimating $\left(\lambda_{\eta}, \lambda_{w}, \rho\right)$ via the posterior mean or via maximum likelihood. Conditional on these parameters, the model output could be emulated by using the mean in Equation (12). Also, we opted to build the emulator over the 12 -d parameter space $C$, requiring the DFT model to predict at any $(Z, N)$, rather than emulate over this extended $12+2$-d space. This was decided because we expected less continuity across the $(Z, N)$ space. However, assessing the relative strengths and weaknesses of alternative emulators is not well studied for this application. Our EOF-based GP emulator is sufficiently accurate for this application; 

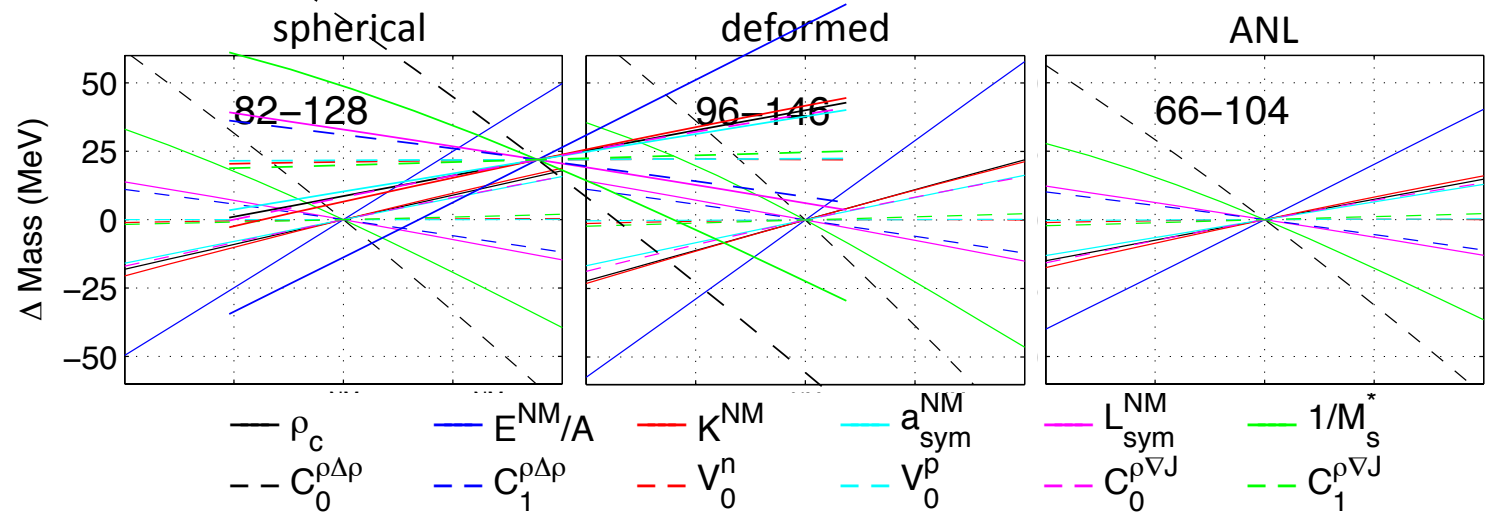

Figure 7. Sensitivity of the masses computed via DFT as each of the 12 parameters is varied from low to high (for ranges see Table 1). The plots show how computed masses change for three nuclei (Z-N) - one spherical, one deformed, and one from the new ANL measurements - as the parameters are varied, one at at time.

testing against holdout DFT runs for randomly chosen $t \in C$ yields a root mean square error of 0.14 and $0.17 \mathrm{MeV}$ for spherical and deformed masses respectively.

\subsection{Full Bayesian GP-based Formulation - Single Output Type}

We now describe the model formulation that incorporates experimental measurements to constrain uncertainty regarding the DFT parameter vector $\theta$. We focus on the spherical mass data and emulator; in the following subsection, we describe how the multiple data types can be combined in a common formulation. Hence we use $n$ to denote the number of experimental measurements, $m$ to denote the number of model runs for each nucleus, and $\left(\lambda_{y}, \lambda_{\eta}, \lambda_{w}, \rho\right)$ to denote the model parameters specific to this data type, leaving the distinction between data types to be made in the following subsection.

We have mass measurements for $n=28$ spherical nuclei held in the $n$-vector $y$. Although these measurements are accurate, we expect errors $\epsilon$ between the measurements and the DFT code, even at the (unknown) best setting $\theta$, giving

$$
y=\eta(\theta)+\epsilon,
$$

where the errors are modeled as $N\left(0, \Sigma_{y}\right)$. We define $\Sigma_{y}=\lambda_{y}^{-1} I_{n}$ and specify a diffuse $G a\left(a_{y}=1, b_{y}=.005\right)$ prior for $\lambda_{y}$, allowing the experimental measurements to inform about these precisions. Using the basis representation for $\eta(\cdot)$ in (1), we obtain a normal-gamma form for the data model

$$
y \mid w(\theta), \lambda_{y} \sim N\left(\Phi w(\theta), \Sigma_{y}\right), \quad \lambda_{y} \sim G a\left(a_{y}, b_{y}\right)
$$

We can now write out the entire posterior distribution for all the parameters, 
including $\theta$. First, let

$$
\begin{aligned}
\hat{w}_{y} & =\left(\Phi^{\prime} \Sigma_{y}^{-1} \Phi\right)^{-1} \Phi^{\prime} \Sigma_{y}^{-1} y \\
a_{y}^{*} & =a_{y}+\frac{1}{2}(n-q) \\
W_{y} & =\lambda_{y} I_{n} \\
b_{y}^{*} & =b_{y}+\frac{1}{2}\left(y-\Phi \hat{w}_{y}\right)^{\prime} W_{y}\left(y-\Phi \hat{w}_{y}\right), \\
\Lambda_{y} & =\Phi^{\prime} \Sigma_{y}^{-1} \Phi \\
\Lambda_{\eta} & =\lambda_{\eta} \Phi^{\prime} \Phi, \\
\Sigma_{w_{y}} & =\operatorname{diag}\left(\lambda_{w_{1}}^{-1}, \ldots, \lambda_{w_{q}}^{-1}\right), \\
\Sigma_{w_{y} w} & =\left(\begin{array}{ccc}
\lambda_{w 1}^{-1} R\left(\theta, \boldsymbol{t}^{*} ; \rho_{1}\right) & 0 & 0 \\
0 & \ddots & 0 \\
0 & 0 & \lambda_{w q}^{-1} R\left(\theta, \boldsymbol{t}^{*} ; \rho_{q}\right)
\end{array}\right)
\end{aligned}
$$

where $R\left(\theta, \boldsymbol{t}^{*} ; \rho\right)$ is the $1 \times m$ matrix with elements $R\left(\theta, t_{j}^{*} ; \rho\right)$,

$$
\begin{aligned}
\hat{z} & =\left(\begin{array}{c}
\hat{w}_{y} \\
\hat{w}
\end{array}\right), \\
\Sigma_{\hat{z}} & =\left(\begin{array}{cc}
\Lambda_{y}^{-1} & 0 \\
0 & \Lambda_{\eta}^{-1}
\end{array}\right)+\left(\begin{array}{cc}
\Sigma_{w_{y}} & \Sigma_{w_{y} w} \\
\Sigma_{w_{y} w}^{\prime} & \Sigma_{w}
\end{array}\right) .
\end{aligned}
$$

The posterior distribution has the form

$$
\begin{aligned}
& \pi\left(\lambda_{\eta}, \lambda_{w}, \rho, \lambda_{y}, \theta \mid \hat{z}\right) \propto \\
& \left|\Sigma_{\hat{z}}\right|^{-\frac{1}{2}} \exp \left\{-\frac{1}{2} \hat{z}^{\prime} \Sigma_{\hat{z}}^{-1} \hat{z}\right\} \times \lambda_{\eta}^{a_{\eta}^{*}-1} e^{-b_{\eta}^{*} \lambda_{\eta}} \times \prod_{i=1}^{q} \lambda_{w i}^{a_{w}-1} e^{-b_{w} \lambda_{w i}} \times \\
& \prod_{i=1}^{q} \prod_{k=1}^{p} \rho_{i k}^{a_{\rho}-1}\left(1-\rho_{i k}\right)^{b_{\rho}-1} \times \prod_{i=1}^{2} \lambda_{y i}^{a_{y}^{*}-1} e^{-b_{y}^{*} \lambda_{y i}} \times I[\theta \in C],
\end{aligned}
$$

where $C$ denotes the 12-dimensional rectangle given in Table 1 and shown in Figure 3.

\subsection{Full Bayesian GP-Based Formulation - Combining Multiple Output Types}

The posterior density in (16) captures the parameter uncertainty resulting from combining the spherical mass measurements with our statistical model formulation. This posterior has the general form

$$
\left\{L\left(\hat{z} \mid \lambda_{\eta}, \lambda_{w}, \rho, \lambda_{y}, \theta\right) \cdot \pi\left(\lambda_{\eta}\right) \cdot \pi\left(\lambda_{w}\right) \cdot \pi(\rho) \cdot \pi\left(\lambda_{y}\right)\right\} \cdot \pi(\theta),
$$

where the terms within the braces are specific to this particular data type. We can derive similar posteriors for additional data types. These multiple data types can be combined to inform about $\theta$ by taking the product of the terms within the brackets

for each data type. Hence information from $K$ data types could be combined with the posterior

$$
\left.\prod_{k=1}^{K}\left\{L\left(\hat{z}^{(k)}\right) \mid \lambda_{\eta}^{(k)}, \lambda_{w}^{(k)}, \rho^{(k)}, \lambda_{y}^{(k)}, \theta\right) \cdot \pi\left(\lambda_{\eta}^{(k)}\right) \cdot \pi\left(\lambda_{w}^{(k)}\right) \cdot \pi\left(\rho^{(k)}\right) \cdot \pi\left(\lambda_{y}^{(k)}\right)\right\} \cdot \pi(\theta),
$$


where the superscript ${ }^{(k)}$ indexes the data type. This product form assumes independence between error terms from different data sources.

Realizations from the posterior distribution (17) are produced by using standard, single-site MCMC. Metropolis updates [26] are used for the components of $\rho$ and $\theta$ with a uniform proposal distribution centered at the current value of the parameter. The precision parameters $\lambda_{\eta}, \lambda_{w}$, and $\lambda_{y}$ are sampled by using Hastings updates [27]. Here the proposals are uniform draws, centered at the current parameter values, with a width that is proportional to the current parameter value. We tune the candidate proposal width for good Monte Carlo efficiency.

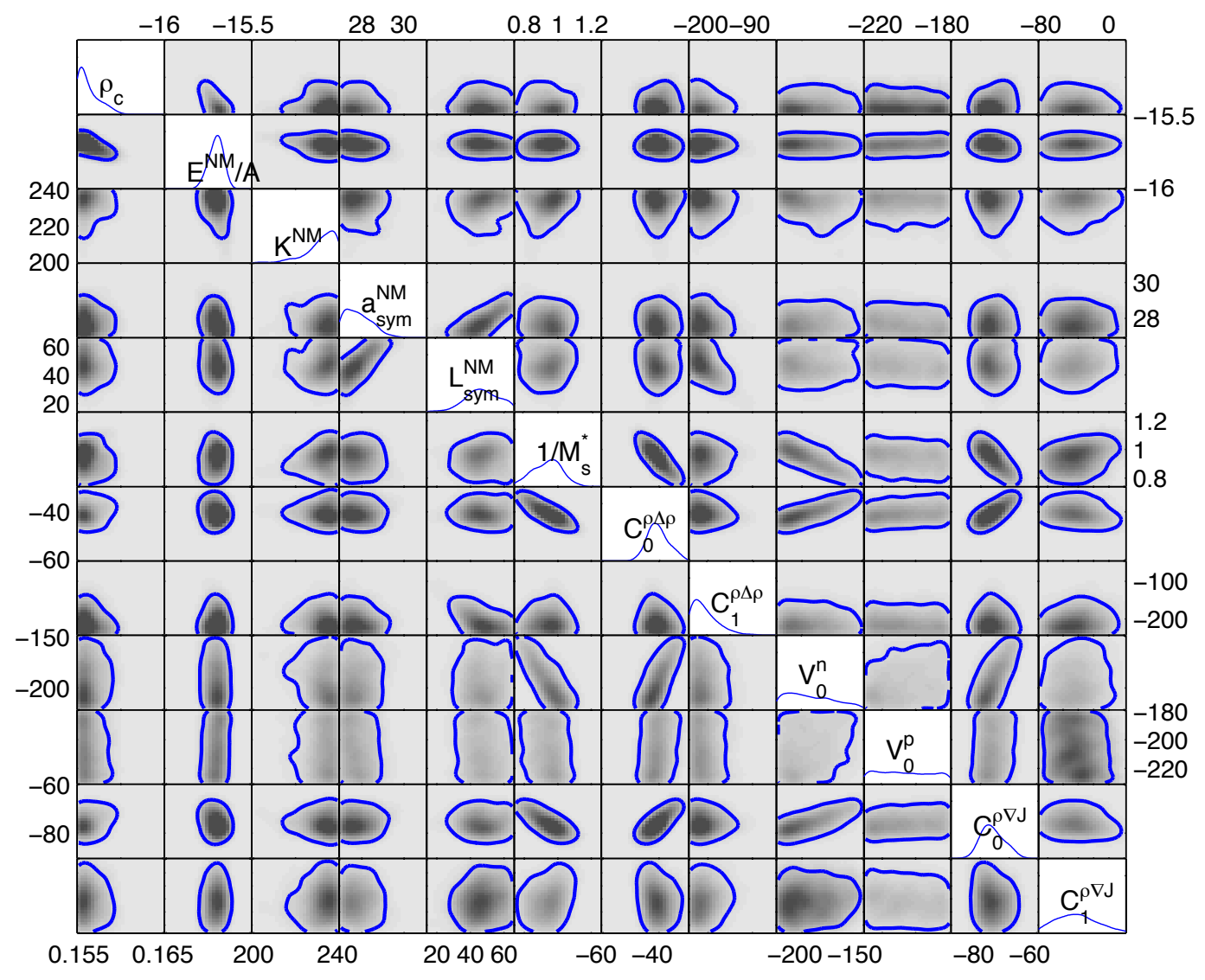

Figure 8. Univariate and bivariate marginal estimates of the posterior distribution for the 12-dimensional DFT parameter vector $\theta$, conditioning on mass measurements from the spherical and deformed nuclei. The blue line encloses an estimated $95 \%$ region.

\section{Posterior Results}

The resulting posterior distribution estimate for $\theta$ is shown in Figure 8 on the original scale. The posterior values can also be propagated through the emulator to produce realizations of the model predictions. All the predictions are centered at the nominal DFT prediction using the parameter setting $t^{0}$ given in [11]. Thus the figures show the difference: prediction $-\eta\left(t^{0}\right)$. Figure 9 shows $90 \%$ prediction intervals for masses of the 
spherical and deformed nuclei used in this formulation. The dark blue bands show $90 \%$ intervals for $\eta(\theta)$ - the result of propagating uncertainty in $\theta$ through the emulator, along with uncertainty in the emulator. The light blue bands show $90 \%$ prediction intervals for the actual measured value $\eta(\theta)+\epsilon$. This prediction also includes the effect of uncertainty in $\epsilon$ - the error between $\eta(\theta)$ and $y$. The magnitude of these errors is controlled by $\lambda_{y}$, which differs for the two data types.
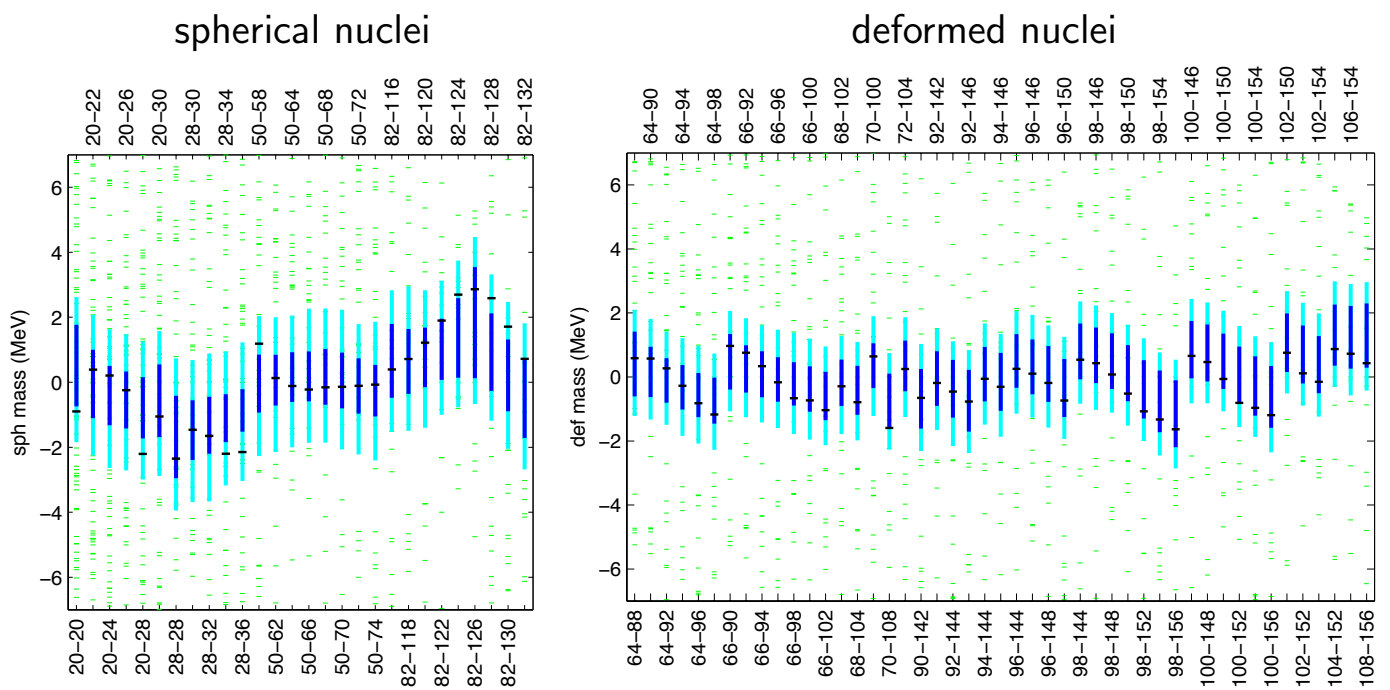

Figure 9. Posterior predictions for the masses of the spherical and deformed nuclei used in the UNEDF1 parametrization. The blue intervals correspond to $\eta(\theta)$; the light blue intervals correspond to $\eta(\theta)+\epsilon$. DFT model output is represented by the green dashes, and experimental measurements by the black dashes. Each nucleus is labeled Z-N.

Comparing the posterior predictions, along with their uncertainty, with the actual mass measurements gives an idea of how well the statistical formulation models the experimental measurements. The mass predictions are more accurate for the deformed nuclei. The dark blue parameter uncertainty bands for $\eta(\theta)$ contain all the experimental measurements, and the estimated standard deviation of $\epsilon$ is about $0.8 \mathrm{MeV}$. For the spherical nuclei, 7 of the 28 measurements are not contained in the $90 \%$ prediction bands for $\eta(\theta)$, and the estimated standard deviation of $\epsilon$ is about $1.3 \mathrm{MeV}$. This larger standard deviation for $\epsilon$ is required in order to make the experimental measurements of spherical nuclei compatible with the statistical model formulation. This difference in prediction quality is primarily because the DFT model more accurately predicts the experimentally measured masses for deformed nuclei.

Using the posterior distribution resulting from this analysis, we can predict the outcome of the ANL mass measurements. The posterior distribution for $\eta(\theta)^{(3)}$ for the calibrated model can be obtained by propagating the posterior draws for $\theta$ through the ANL mass emulator. The resulting 90\% intervals are given by the blue intervals in Figure 10. Predictions for the new measurements $\eta^{(3)}(\theta)+\epsilon^{(3)}$ require the variance, or a probabilistic description about the variance of $\epsilon^{(3)}$ - the model error for the new ANL 
predictions. For the predictions in Figure 10 we classified each of the ANL nuclei as spherical or deformed, and we assigned the appropriate precision estimate $\lambda_{y}$ obtained from the spherical and deformed nuclei accordingly. Since the first 4 nuclei are spherical, their prediction uncertainty is slightly larger than that of the remaining 13 deformed nuclei. The resulting $90 \%$ intervals are given by the light blue lines in Figure 10.

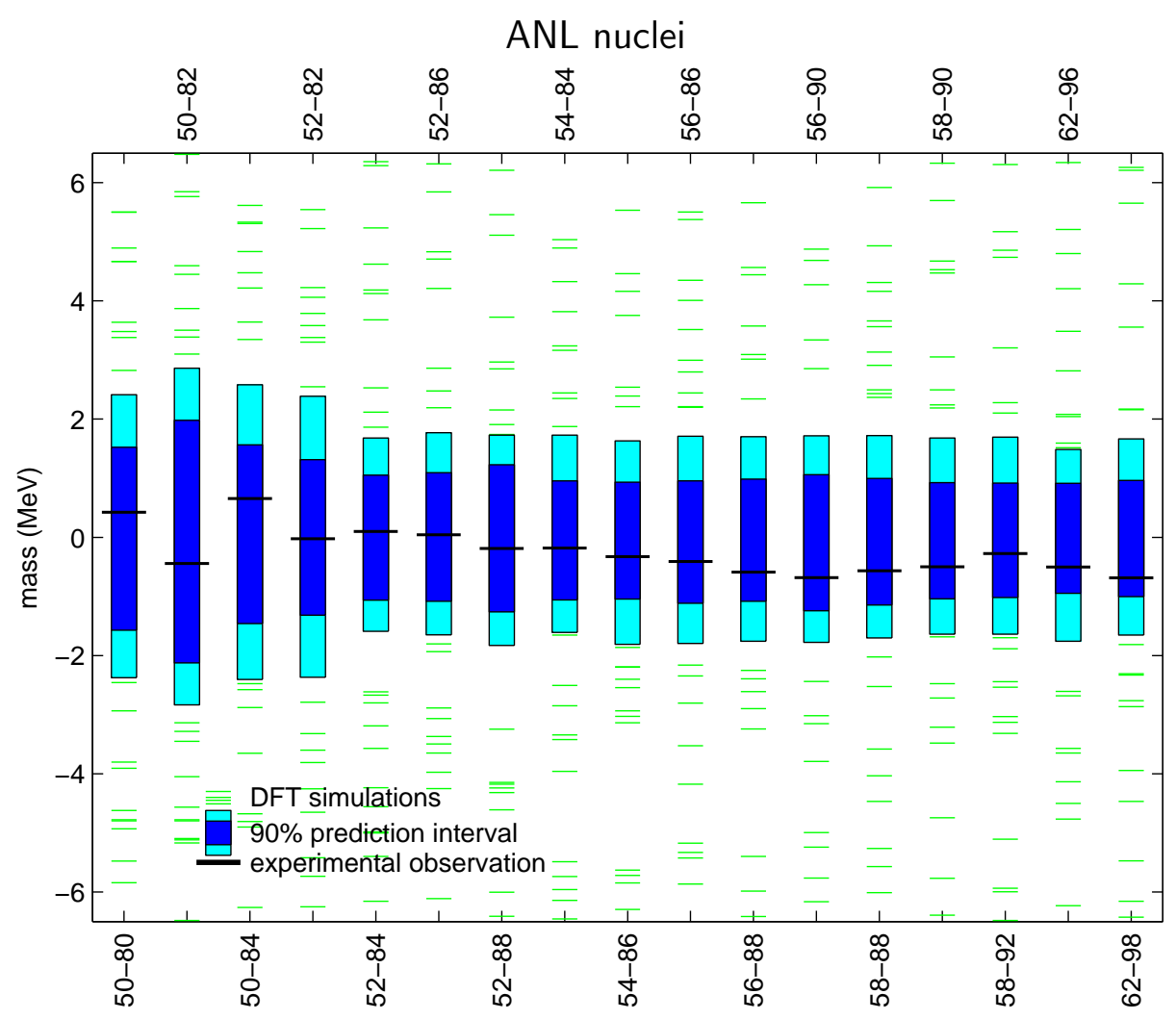

Figure 10. Posterior predictions for the 17 new ANL masses. The blue intervals correspond to $\eta(\theta)$; the light blue intervals correspond to $\eta(\theta)+\epsilon$. These predictions used only data from the 28 spherical and 47 deformed mass measurements used in UNEDF1. DFT model output is represented by the green dashes, experimental measurements by the black dashes. Each nucleus is labeled Z-N.

\section{Discussion}

The purpose of this paper is to describe the Bayesian model calibration approach in detail, focusing on an example in DFT-based modeling and prediction. While we have treated this statistical analysis with care, paying particular attention to the scientific issues, this analysis is not meant to be definitive in a scientific sense. A more scientifically focused analysis is given by Schunck et al. [9] in this issue.

An important feature of this analysis is the impact of additional experimental data on the analysis. The prediction for new measurements is given by $\eta(\theta)+\epsilon$. In general, more data reduces the uncertainty regarding the model parameters $\theta$, reducing the uncertainty in the calibrated model $\eta(\theta)$. However, it will not strongly impact the 
standard deviation of $\epsilon$. Hence, to produce realistic prediction uncertainties, one must accurately characterize the uncertainty in the model error term $\epsilon$.

How much the uncertainty in $\theta$ can be reduced by a particular type of data depends on the DFT model $\eta(\cdot)$. Figure 7 clearly shows that mass data alone will not reduce uncertainty in certain linear combinations of the model parameters. For example, moving $1 / M_{s}^{*}$ and $C_{0}^{\rho \Delta \rho}$ together will not have a large impact on the DFT-computed mass. The reason is that the posterior uncertainty regarding these two parameters is strongly correlated. Because of this similarity in sensitivity, additional mass data will not substantially improve this situation.

This analysis estimated the error variances, allowing different precision parameters $\lambda_{y}$ for the spherical and deformed masses. This approach results in a posterior distribution for $\theta$ that gives more accurate results for deformed nuclei, relative to the spherical ones. In contrast, previous analyses $[10,11]$ have given equal weight to the two data types. There the weights $w$ in the objective function effectively specify the precision $\lambda_{y}$ for each data type $\left(w=\lambda_{y}^{-\frac{1}{2}}\right)$, leading to a different posterior distribution for $\theta$.

\section{Acknowledgment}

This material was based upon work supported by the U.S. Department of Energy, Office of Science, Advanced Scientific Computing Research SciDAC program. Computational resources were provided through an INCITE award "Computational Nuclear Structure" by the National Center for Computational Sciences (NCCS) and National Institute for Computational Sciences (NICS) at Oak Ridge National Laboratory, through an award by the Livermore Computing Resource Center at Lawrence Livermore National Laboratory, and through an award by the Laboratory Computing Resource Center at Argonne National Laboratory.

\section{References}

[1] M. Kennedy and A. O'Hagan. Bayesian calibration of computer models (with discussion). J. R. Stat. Soc. Series B, 68:425-464, 2001.

[2] D. Higdon, M. Kennedy, J. Cavendish, J. Cafeo, and R. D. Ryne. Combining field observations and simulations for calibration and prediction. SIAM J. Sci. Comput., 26:448-466, 2004.

[3] M. J. Bayarri, J. O. Berger, R. Paulo, J. Sacks, J. A. Cafeo, J. Cavendish, C. Lin, and J. Tu. A framework for validation of computer models. Technometrics, 49:138-154, 2007.

[4] J. P. Kaipio and E. Somersalo. Statistical and Computational Inverse Problems. Springer, New York, 2004.

[5] A. Tarantola. Inverse problem theory and methods for model parameter estimation. SIAM, 2005.

[6] J. Sacks, W. J. Welch, T. J. Mitchell, and H. P. Wynn. Design and analysis of computer experiments (with discussion). Stat. Sci., 4:409-423, 1989.

[7] J. Oakley and A. O'Hagan. Probabilistic sensitivity analysis of complex models. J. R. Stat. Soc. Series B, 66:751-769, 2004.

[8] E. N. Ben-Ari and D. M. Steinberg. Modeling data from computer experiments: An empirical 
comparison of kriging with MARS and projection pursuit regression. Qual. Engin., 19(4):327$338,2007$.

[9] N. Schunck, J. D. McDonnell, J. Sarich, S. M. Wild, and D. Higdon. Error analysis in nuclear density functional theory. Preprint ANL/MCS-P5145-0514, ANL, May 2014.

[10] M. Kortelainen, T. Lesinski, J. Moré, W. Nazarewicz, J. Sarich, N. Schunck, M. V. Stoitsov, and S. M. Wild. Nuclear energy density optimization. Phys. Rev. C, 82(2):024313, 2010.

[11] M. Kortelainen, J. McDonnell, W. Nazarewicz, P.-G. Reinhard, J. Sarich, N. Schunck, M. V. Stoitsov, and S. M. Wild. Nuclear energy density optimization: Large deformations. Phys. Rev. C, 85(2):024304, 2012.

[12] J. Van Schelt, D. Lascar, G. Savard, J. A. Clark, P. F. Bertone, S. Caldwell, A. Chaudhuri, A. F. Levand, G. Li, G. E. Morgan, et al. First results from the CARIBU facility: Mass measurements on the r-process path. Phys. Rev. Lett., 111(6):061102, 2013.

[13] D. Gamerman and H. F. Lopes. Markov chain Monte Carlo: Stochastic simulation for Bayesian inference. CRC Press, 2006.

[14] W. J. Welch, R. J. Buck, J. Sacks, H. P. Wynn, T. J. Mitchell, and M. D. Morris. Screening, predicting, and computer experiments. Technometrics, 34:15-25, 1992.

[15] A. O'Hagan. Bayesian analysis of computer code outputs: A tutorial. Rel. Engin. Es Sys. Safety, 91(10):1290-1300, 2006.

[16] K. Heitmann, D. Higdon, S. Habib, and C. Nakhleh. Cosmic calibration. Astrophys. J. Lett., 646:L1, 2006.

[17] B. Sanso, C.E. Forest, and D. Zantedeschi. Inferring climate system properties using a computer model. Bayesian Analysis, 3(1):1-38, 2008.

[18] B. Tang. Orthogonal array-based Latin hypercubes. J. Am. Stat. Assoc., 88:1392-1397, 1993.

[19] T. J. Santner, B. J. Williams, and W. I. Notz. Design and analysis of computer experiments. Springer, New York, 2003.

[20] Jonathan Rougier. Efficient emulators for multivariate deterministic functions. J. Comp. Graph. Statis., 17(4):827-843, 2008.

[21] M.J. Bayarri, J.O. Berger, E. Calder, K. Dalbey, S. Lunagomez, A.K. Patra, B.E. Pitman, E.T. Spiller, and R.L. Wolpert. Using statistical and computer models to quantify volcanic hazards. Technometrics, 51(4), 2009.

[22] D. Higdon, J. Gattiker, B. Williams, and M. Rightley. Computer model calibration using highdimensional output. J. Am. Stat. Assoc., 103(482):570-583, 2008.

[23] J. O. Ramsay and B. W. Silverman. Functional Data Analysis. Springer, New York, 1997.

[24] H. von Storch and F. W. Zwiers. Statistical Analysis in Climate Research. Cambridge University Press, New York, 1999.

[25] J. Besag, P. J. Green, D. M. Higdon, and K. Mengersen. Bayesian computation and stochastic systems (with discussion). Stat. Sci., 10:3-66, 1995.

[26] N. Metropolis, A. Rosenbluth, M. Rosenbluth, A. Teller, and E. Teller. Equations of state calculations by fast computing machines. J. Chem. Phys., 21:1087-1091, 1953.

[27] W. K. Hastings. Monte Carlo sampling methods using Markov chains and their applications. Biometrika, 57:97-109, 1970. 